\title{
Recombinant hepatitis B virus core particles: association, dissociation and encapsidation of green fluorescent protein.
}

\begin{abstract}
The recombinant hepatitis B virus (HBV) core antigen ( $\mathrm{HBcAg}$ ) expressed in Escherichia coli self-assembles into icosahedral capsids of about $35 \mathrm{~nm}$ which can be exploited as gene or drug delivery vehicles. The association and dissociation properties of the C-terminally truncated $\mathrm{HBcAg}$ with urea and guanidine hydrochloride $(\mathrm{GdnHCl})$ were studied. Transmission electron microscopy (TEM) revealed that the dissociated HBcAg was able to re-associate into particles when the applied denaturing agents were physically removed. In order to evaluate the potential of the particles in capturing molecules, purified green fluorescent protein (GFP) was applied to the dissociated $\mathrm{HBcAg}$ for encapsidation. The $\mathrm{HBcAg}$ particles harbouring the GFP molecules were purified using sucrose density gradient ultracentrifugation and analysed using native agarose gel electrophoresis and TEM. A method for the encapsidation of GFP in HBcAg particles which has the potential to capture drugs or nucleic acids was established.
\end{abstract}

Keyword: Hepatitis B virus capsid; Denaturing agent; Capsid assembly; Hepatitis B core antigen; Green fluorescent protein; Protein encapsidation. 